\title{
Derecho a la educación superior
}

\section{Right to higher education}

\author{
Claudia Hilda Paparini \\ https://orcid.org/0000-0002-8004-6054 \\ cpaparini@uncu.edu.ar \\ Universidad Nacional de Cuyo I Argentina
}

\section{RESUMEN}

Los derechos humanos se constituyen en la garantía jurídica universal para una vida libre y digna. La legislación obliga a los Estados, principalmente y a otros agentes como responsables para su efectivización y para actuar frente a los impedimentos que dificultan e impiden su realización. Su reconocimiento formal es una realidad que contrasta con las situaciones de discriminación y exclusión que se verifican en diversas situaciones particulares. La ampliación y efectiva concreción del derecho a la educación superior no escapa a la problemática señalada. Con reconocimiento formal en la normativa internacional, regional y nacional, la problemática se hace evidente en las diversas instituciones de educación superior.

El artículo pretende reflexionar sobre algunos lineamientos de la teoría crítica en vistas a garantizar el derecho a la educación superior. Se centra en el análisis del "habitus institucional" como uno de los factores claves para que las instituciones de nivel superior ejerzan su responsabilidad como agentes claves en su garantía. Finalmente, refiere al proyecto institucional de carácter preventivo del fracaso académico desarrollado en la Universidad Nacional de Cuyo (UNCuyo) denominado Trayectorias Académicas Estudiantiles (TRACES).

\section{ABSTRACT}

Human rights constitute the universal legal guarantee for a free and dignified life. The legislation obliges mainly States, and other agents, as the ones responsible for its realization and to act against the impediments that hinder and impede its realization. Its formal recognition is a reality that contrasts with the situations of discrimination and exclusion that are verified in various particular situations. The extension and effective realization of the right to university education does not escape the aforementioned problem. With formal recognition in international, regional and national regulations, the problem becomes evident in the various institutions of university education.

The article aims to reflect on some guidelines of the critical theory in order to guarantee the right to university education. It focuses on the analysis of the "institutional habitus" as one of the key factors for university level institutions to exercise their responsibility as a key agent in their guarantee. Finally, it refers to the institutional project of preventive nature of academic failure developed at the National University of Cuyo (UNCuyo) called Student Academic Career (TRACES).

PALABRAS CLAVE

Derechos humanos, derecho a la educación superior- habitus institucional- trayectorias académicas estudiantiles.

\section{KEY WORDS}

human rights- right to university educationinstitutional habitusstudent's academic career 


\section{DERECHOS HUMANOS, UNIVERSALES PERO...}

La Organización de las Naciones Unidas (ONU), aprueba la Declaración de los Derechos Humanos el 10 de diciembre de 1948. La Proclama reconoce la dignidad intrínseca de todos los seres humanos y establece derechos iguales e inalienables de todos como una condición para alcanzar una vida más digna y justa.

Desde entonces, numerosos Pactos y Conferencias Internacionales, que no tiene objeto citar en este trabajo, han intentado ampliar y precisar las condiciones de dignidad de las personas. A su vez, se ha avanzado a través de distintas convenciones o tratados destinados a brindar protección a ciertos grupos de personas -apátridas, mujeres, niños, trabajadores- $y$ a ciertas ofensas o delitos especialmente graves contra los derechos humanos -trata de personas, discriminación racial, genocidio, tortura, desaparición forzada de personas.

La relación entre democracia y derechos humanos se concreta en el plano jurídico a través de las Constituciones y leyes que los detaIlan y protegen. No hay democracia sin una base en principios éticos fundamentales que garanticen derechos y que impongan un conjunto de deberes. La democracia se constituye en una condición indispensable para la vigencia de los derechos. En esta dirección, Boaventura de Sousa Santos nos advierte que"Ios derechos humanos de baja intensidad aparecen como la otra cara de la democracia de baja intensidad" (2010: 71).

Se torna imposible desconocer el alcance político y moral que ha logrado el lenguaje universalista de los derechos humanos. "La expansión de los derechos humanos como lenguaje hegemónico sobre la dignidad humana parece incuestionable" (Sánchez Rubio, 2015: 183). 
A pesar del éxito discursivo, la marginación y discriminación por razones sociales, culturales, raciales, étnicas, etarias, territoriales, educativas se incrementa estructuralmente. Discurso hegemónico que "incluye" en abstracto, pero alejado de las prácticas sociales concretas en tanto un gran porcentaje de la población no es considerado como sujeto de derechos.

Se torna muy difícil que las normas jurídicas y las instituciones estatales puedan confrontar y transformar las asimetrías y desigualdades estructurales, entre otras razones porque las situaciones de injusticia se invisibilizan en lo cotidiano. Los modos de dominación en las relaciones sociales cotidianas y a nivel institucional naturalizan estas formas de desigualdad y opresión. En esta línea de análisis, Boaventura de Sousa Santos destaca la necesidad de distinguir concepciones hegemónicas de los derechos humanos que tienden a "legitimar las ideologías del individualismo propietario $y$, en consecuencia, a reproducir el (des)orden social capitalista, colonialista y patriarcal que domina nuestro tiempo" de las nociones contrahegemónicas que promueven "alternativas de autonomía" (2014:43) en el marco de la creación de una sociedad más digna y justa.

Desde esta perspectiva, la ambición universalista de los derechos humanos se construye sobre la base de estructuras de dominación de unas jerarquías sociales, económicas, políticas, culturales, geográficas y epistémicas por sobre otras, obturando así, la posibilidad de contar con las condiciones de una vida digna para todos en todas sus dimensiones. Sánchez Rubio (2015:189) resalta que: "Occidente establece una sociabilidad de inclusiones abstractas sobre la base de exclusiones concretas y cotidianas".

En este sentido y dando continuidad al razonamiento anterior, el sujeto de derechos es un sujeto abstracto, vacío, sin identidad, sin contexto, sin historia, sin condicionantes. En general, este individuo abstracto encarna en el individuo "blanco, varón, mayor de edad, propietario, emprendedor, creyente religioso cristiano, heterosexual, competitivo e individualista" (Sánchez Rubio,2015: 197). Esta situación cobra tal preeminencia que deja sin derechos a la mayoría que no cumple con esos atributos (mujeres, homosexuales, negros, pobres, pueblos indígenas, etc.).

La inclusión, de este modo, se sostiene en una idea abstracta y formal de igualdad y no, en el reconocimiento de las desigualdades y diferencias de muchas personas para las que la situación de menosprecio, opresión y explotación en la que viven, poco tiene que ver con el respeto por la dignidad humana. La noción universal de igualdad que da lugar a la definición de derechos inalienables e inherentes a la persona tendría que ser sistemáticamente cuestionada desde la cotidianeidad de nuestras prácticas sociales.

Problematizar la matriz colonial de los derechos humanos supone historizarlos, visibilizar su desarrollo en lo particular, situarlos para reconocer los procesos que operan a través de distintos modos de dominación y que culminan en la clasificación de humanos superiores e in- 
feriores. De lo contrario, las tramas sociales y relacionales desiguales y asimétricas del saber, del poder, del ser, del tener y del hacer seguirán obsturando la generación de condiciones para que los mismos sean disfrutados por la mayoría de la humanidad. Las relaciones construidas desde este imaginario hegemónico, con aspiraciones universalistas han sido construidas simbólicamente desde esta matriz colonial.

En este punto, se estima indispensable recurrir a la teoría crítica de los derechos humanos para visibilizar fenómenos, denunciar las injusticias, cuestionarlas, problematizarlas, y habilitar la intervención individual y colectiva se torna indispensable.

La teoría crítica cumple, en primer lugar, con la función epistémica que permita visibilizar las relaciones sociales existentes, que permita "hacer visible lo invisible" (Foucault) en referencia a aquellas realidades que apenas podemos percibir por estar tan cercanas a nosotros. Encontrarse con la desigualdad y las diferencias invisibilizadas y silenciadas es el primer paso para acercarse al acto transformador. La segunda función es ética, poniendo en crisis, desestabilizando, cuestionando lo que ocurre, lo que se da por evidente y natural. El pensar de "otro modo" se concreta enriqueciendo el análisis de los contextos. La tercera función de la teoría crítica es política, en tanto propone acciones y procesos emancipadores que transformen la realidad.

No se trata de tres funciones que sigan un orden cronológico en la labor del teórico; no constituyen etapas de la teoría, sino dimensiones del acto teorizador. No hay una mirada neutra, desprendida de una postura ética e ideológica y desprovista de opciones políticas. Se desestabiliza desde la apuesta por un determinado proyecto de sociedad, o al menos desde la conciencia de la necesidad de superar el existente. Sólo se transforma desde esa mirada "otra", alternativa, desde esa pretensión de movilizar para potenciar, para abrir nuevas posibilidades.

Joaquín Herrera Flores insta a recuperar lo real a través de la "lectura sintomática" que visibilice las rupturas, contradicciones y discontinuidades, y potenciar otras formas a través del desarrollo de prácticas instituyentes de un nuevo orden social. El pensamiento crítico es siempre creativo y afirmativo". (2008: 55). Así también lo destaca cuando especifica que la teoría crítica debe asumir tres compromisos:

1. Desenterrar continua y permanentemente lo que queda olvidado/ocultado

2. Establecer de un modo constante relaciones y vínculos que han sido negados, y

3. Señalar recurrentemente cursos alternativos de acción social y de reflexión intelectual. (Herrera Flores, J; 2005: 187)

La teoría crítica de los derechos humanos requiere que el pensamiento asuma el proceso de continua reconstrucción y no quede protegido al amparo de ideas dogmáticas. Por el contrario, el pensamiento crítico debe vivir inmerso en las prácticas sociales, dialogar, buscar 
alternativas, problematizar las relaciones, pero sobre todo, cuestionarse a sí mismo. El reto consiste en buscar nuevas bases epistémicas que permitan pensar, repensar, pensar distinto, superar cualquier forma de dogmatismo y buscar prácticas emancipatorias alternativas al orden/ desorden existente.

\section{El reto consiste en buscar nuevas bases epistémicas que permi- tan pensar, repensar, pensar distinto, superar cualquier forma de dogmatismo y buscar prácticas emancipatorias alternativas al orden/desorden existente.}

\section{LA EDUCACIÓN SUPERIOR COMO DERECHO HUMANO}

Las Conferencias Regionales y Mundiales de Educación Superior desarrolladas desde 1996 han contribuido al debate y consolidación de la democratización del Nivel Superior, especialmente a escala Regional. La consideración de la Educación Superior como bien público social, un derecho humano y universal y su garantía, un deber de los Estados ha influido sin dudas en el desarrollo de un rico marco normativo a nivel de los Estados Nacionales.

En nuestro caso, la Ley 26.206 de Educación Nacional (2006) legisla el derecho a la educación como bien público y un derecho personal y social, garantizado por el Estado. La Ley 24.521 de Educación Superior a partir de su modificación en noviembre de 2015 (Ley N²7.204) reconoce a la educación y al conocimiento como bien público y derecho humano personal y social, y garantiza el carácter público y gratuito de la enseñanza superior.

A nivel local, es importante señalar que el Estatuto de la Universidad Nacional de Cuyo, reformado en 2013 reconoce a la educación como bien público, gratuito y laico, como derecho humano y como obligación del Estado.

En términos regionales se puede dar cuenta del incremento de las instituciones y de la matrícula del Nivel Superior, pero también debemos reconocer que este crecimiento ha sido segmentado y con un alto grado de diferenciación institucional. Fenómeno denominado "expansión condicionada" por Pablo Gentili (2009) para aludir al crecimiento con diversificación y fragmentación de la oferta de estudios a través de instituciones y circuitos formativos universitarios de distinta calidad académica.

El éxito discursivo respecto del reconocimiento del derecho a la educación superior a nivel Regional, Nacional e Institucional se ve contrastado por innumerables situaciones que dan cuenta del fracaso en las prácticas particulares, en tanto un gran porcentaje de nuestros estudiantes no son reconocidos como sujetos del derecho a la educación superior. La marginación y discriminación por razones sociales, culturales, raciales, étnicas, etarias, territoriales, educativas atraviesa el 
subsistema y a las propias instituciones. Problema complejo y multidimensional, que requiere de políticas específicas para garantizar su realización.

Sin dudas, aspectos relacionados con la distribución de la riqueza y del capital cultural son aspectos altamente condicionantes de las oportunidades y posibilidades de muchos estudiantes para ejercer este derecho. Sin atribuir toda la responsabilidad a las instituciones universitarias, no podemos dejar de señalar que el campo institucional es un agente clave para comprender la configuración de las experiencias educativas. Se focaliza en lo institucional como un lugar de encuentro entre la enunciación universal del derecho y la realización particular del mismo. Y fundamentalmente, porque es un nivel en el que se cuenta con posibilidades de intervenir, de transformar desde el replanteo de las prácticas educativas.

La matriz fundacional de gran parte de las instituciones universitarias está ligada a la diferenciación y selección. A lo largo del tiempo, muchas universidades no se han pensado como garantes del derecho a la educación superior, no han interpelado y desafiado sus prácticas desde perspectivas basadas en el derecho humano a la educación. Por el contrario, la selección que excluye se ha constituido como uno de los valuartes de reconocimiento académico.

En este punto, apelamos a la categoría "habitus institucional" (Mc Donough,1996; Reay, 1998, 2001) como posibilidad para teorizar en forma crítica -según la propuesta de Herrera Flores- sobre las prácticas en instituciones educativas. Se estima que este concepto se convierte en una herramienta teórica y metodológica clave pues articula fuertemente entre los procesos macro (universales) y micro (particulares). Refiere específicamente a las predisposiciones, apreciaciones, esquemas de percepción y acción sobre los que se organizan las instituciones y que juegan un rol central en la explicación de las oportunidades de los estudiantes en una institución particular. Los diferentes habitus institucionales crean diferentes marcos de posibilidad para la realización del derecho a la educación superior.

La noción de habitus institucional no refiere a un ente unificado, por el contrario, es un campo atravesado por múltiples contradicciones y ambigüedades por lo que pueden emerger múltiples disposiciones dentro el mismo habitus institucional. Asimismo, y como ya se afirmara, puede transformarse, es susceptible de modificarse a partir de su desnaturalización, de su problematización para favorecer la trayectoria académica de los estudiantes.

Como el "habitus" individual implica una combinación de estructura y agencia. En efecto, Bourdieu identifica en esta categoría un conjunto de disposiciones que integran las experiencias pasadas y funcionan en cada momento, como una matriz que condiciona las percepciones, apreciaciones y prácticas a futuro. Hablar de habitus implica, por un lado, atender a las experiencias del pasado y por otro, preformar otras prácticas futuras tendiendo a evitar la reiteración y reproducción de la experiencia pasada. 
El concepto pretende atender a las lógicas subyacentes, las razones por las cuales las instituciones hacen lo que hacen, atender al cómo y por qué hacen lo que hacen. Se trata de desentrañar y reflexionar sobre las microdinámicas a partir de las cuales se configuran y desarrollan las prácticas educativas en cada institución educativa. Supone preguntarse por las percepciones y creencias colectivas acerca de los estudiantes, de la educación y de la institución en sí misma. El propósito es desentrañar, reflexionar y transformar las dinámicas de segregación y discriminación naturalizadas que se dan cotidianamente en las instituciones universitarias.

La pedagogía crítica nunca pude ser pensada como una receta aplicable a cualquier situación. Los principios y prácticas pedagógicas no se pueden aplicar indiscriminadamente a cualquier situación educativa, por el contrario, se definen en contexto. Por el contrario, la pedagogía se delimita en la respuesta a las condiciones, formaciones y problemas de cada espacio-tiempo educativo.

\section{Los principios y prácticas pedagógicas no se pueden aplicar indiscriminadamente a cualquier situación educativa, por el contrario, se definen en contexto.}

\section{PROYECTO TRACES UNCUYO}

El Proyecto Trayectorias Académicas Estudiantiles (TRACES) establece las líneas de acción de la gestión académica 2012-2014 de la Universidad Nacional de Cuyo. Constituyó una propuesta de trabajo en red entre Secretaría Académica de Rectorado, las quince Unidades Académicas y las diversas áreas y servicios de la Universidad, con el propósito de abordar las diferentes problemáticas institucionales que afectan las trayectorias estudiantiles. Aprobado por Ordenanza 23/2012 del Consejo Superior de la UNCuyo, establece como objetivos:

- "Garantizar el derecho a la educación en la UNCuyo a partir de la comprensión de las trayectorias académicas de sus estudiantes, como procesos situados y contextuados, históricamente construidos, múltiples y no lineales y pasibles de ser repensados y modificados, que se organizan en una continuidad articulada y no como momentos aislados unos de otros.

- Desarrollar una propuesta de trabajo en red de acompañamiento a las Trayectorias Académicas Estudiantiles, que propicie un marco de acciones conjuntas en nuestra Universidad y que contemple a la par, y de manera flexible, las diferencias institucionales propias de cada Facultad y/o Instituto de acuerdo con sus necesidades, demandas y experiencias previas construidas, tendientes a la mejora del desempeño académico de los estudiantes". 
El proyecto surgió como producto de la reflexión y análisis colectivo de las acciones desarrolladas por la Universidad en el marco de la inclusión educativa, hasta ese momento preponderantemente de carácter remedial. El propósito focalizó en el análisis de los problemas asociados al ingreso, permanencia y egreso de cada una de las unidades académicas de la UNCuyo. De este modo, se promovió que cada institución, de manera flexible y siempre contextuada, problematice sus prácticas institucionales, su habitus y proponga sus propias líneas de acción destinadas a ampliar el derecho a la educación superior.

\section{El propósito focalizó en el análisis de los problemas asociados al ingreso, permanencia y egreso de cada una de las unidades académicas de la UNCuyo.}

Desde el nivel central se financió el desarrollo de cada uno de los proyectos institucionales tomando en consideración la complejidad de cada unidad académica (cantidad de estudiantes, carreras, sedes, etc.) y las condiciones propias de cada una. Asimismo, se preveía la realización de acciones transversales y comunes a todas las Facultades e Institutos formativas para los equipos de gestión del proyecto como así también jornadas que permitieran poner en común y analizar las experiencias institucionales proyectadas y su evaluación.

El diseño del proyecto establecía que los secretarios académicos de las diversas Facultades e Institutos debían asumir su dirección de modo de favorecer su impacto institucional y que no quedase circunscripto a los servicios de orientación tradicionalmente centrados en el tratamiento de la problemática del rendimiento académico. Precisamente, lo que se pretendía era extender el trabajo pedagógico a la totalidad de la institución. La incorporación de los distintos actores institucionales, especialmente profesores y personal de apoyo docente se basó en la convicción de profundizar la problematización de las prácticas institucionales fuertemente condicionadas por las formas de percibir y actuar de acuerdo a su particular habitus institucional.

De acuerdo con Meirieu (2006:53) la necesidad de "pedagogizar la organización", de problematizar y hacer hacia adentro conjuntamente, favorecería la posibilidad de mirar y repensar las trayectorias académicas de los estudiantes, elaborar propuestas de acción para atender las problemáticas detectadas, y evaluar anualmente su implementación a través de indicadores de rendimiento académico definidos por el citado proyecto. De este modo, la problematización de los propios hábitus institucionales, marcados también por el área disciplinaria de formación, facilitaría la posibilidad de superar la permanente comparación entre trayectorias académicas "ideales" y las "reales".

El análisis de las trayectorias desde la perspectiva institucional supone interpelar de qué forma ese entramado, en continuo movimiento, opera como obstáculo o como condición de posibilidad de un proceso de formación. Focalizar en lo institucional, para incidir en la modificación de 
las diversas dimensiones del habitus que condiciona las prácticas pedagógicas y así, acompañar a los estudiantes en su trayectoria académica.

Comprender las diversas trayectorias académicas de los estudiantes desde la dimensión institucional habilitaría la apertura de espacios para el trabajo colaborativo tendiente a la reflexión y definición de dispositivos pertinentes a las diversas realidades institucionales. Se trata de articular las decisiones sobre aspectos académicos, administrativos, normativos, pedagógicos y formativos en clave particular de cada cultura institucional.

Asimismo, se definieron una serie de indicadores y categorías (permanencia, rendimiento académico, progresión, egreso, etc.) para evaluar los procesos y resultados alcanzados por el desarrollo de cada proyecto tanto a nivel de cada Facultad e Instituto como en conjunto de toda la Universidad. La reflexión sobre los mismos se constituye en la base sobre la cual se definirían las nuevas propuestas institucionales.

La Universidad Nacional como lugar de lo público, de construcción de lo común, supone considerar la singularidad, la especificidad, las condiciones de lo contextual. TRACES constituye una política académica institucional impulsada desde el Rectorado, que pretendió abordar la diversidad de trayectorias académicas de los estudiantes como un continuum a partir de la especificidad de la cultura de las unidades académicas para visibilizar y abordar institucionalmente los obstáculos, desajustes, y contradicciones que inciden en el acceso, permanencia y egreso. Prácticas institucionales sobre las que podemos actuar para transformar y así, construir condiciones de posibilidad que hagan efectiva la ampliación del derecho a la educación superior. Prácticas por las que finalmente, la Universidad como agente del Estado, es responsable. 


\section{BIBLIOGRAFIA}

Abad, Sebastián y Cantarelli, Mariana (2010). "Habitar el Estado". Buenos Aires:

Bourdieu, Pierre (1997). “Razones prácticas. Sobre la teoría de la acción". Barcelona: Anagrama.

De Souza Santos, Boaventura (2010). “Descolonizar el saber, reinventar el poder". Montevideo, Uruguay: CLACSO: Trilce Editorial.

------(2014). “Derechos humanos, democracia y desarrollo". Bogotá: Centro de Estudios de Derecho, Justicia y Sociedad, Dejusticia.

Del Valle, D. y otros (2017). "El derecho a la Universidad en Perspectiva Regional". Buenos Aires, Argentina: CLACSO/IEC-CONADU.

Gentili, P. (2009). "Marchas y contramarchas: el derecho a la educación y las dinámicas de exclusión incluyente en América Latina (a sesenta años de la Declaración Universal de los Derechos Humanos)". Revista Iberoamericana de Educación, no49, pp. 19-57.

Herrera Flores, Joaquín (2008). "La reinvención de los derechos humanos". Andalucía, España: Editorial Atrapasueños.

Herrera Flores, Joaquín. (2005). "Los derechos humanos como productos culturales. Crítica del humanismo abstracto". Madrid: Libros de la Catarata.

Mc Donough, Patricia (1996). “Cómo elegir universidades: cómo se estructuran la clase social y las escuelas". Oportunidad Nueva York: Universidad Estatal de Nueva york.

Meirieu, Phillippe (2006). "Carta a un joven profesor. Por qué enseñar hoy". Barcelona: Editorial GRAÓ.

Nicastro, Sandra y Greco, M. Beatriz (2009). “Entre trayectorias. Escenas y pensamientos en espacios de formación"., Bs. As.: Homo Sapiens.

Sánchez Rubio, D. (2015). “Derechos humanos, no colonialidad y otras luchas por la dignidad: una mirada parcial y situada". Campo Jurídico, 3 (1), pp. 181-213.

Universidad Nacional de Cuyo (2012). Ordenanza CS23/12. Aprobación Proyecto TRACES. Mendoza. 